OPEN ACCESS

Edited by:

Chunhua Bian,

Nanjing University, China

Reviewed by:

Ronny P. Bartsch,

Bar-llan University, Israel

Paul Bogdan,

University of Southern California,

United States

*Correspondence:

Deyu L

deyuli@buaa.edu.cn

Shuyu Li

shuyuli@buaa.edu.cn

Specialty section: This article was submitted to

Fractal Physiology, a section of the journal

Frontiers in Physiology

Received: 23 October 2017

Accepted: 20 April 2018

Published: 09 May 2018

Citation:

Li X, Li Q, Wang X, Li D and Li S (2018) Differential Age-Related

Changes in Structural Covariance Networks of Human Anterior and Posterior Hippocampus.

Front. Physiol. 9:518

doi: 10.3389/fphys.2018.00518

\section{Differential Age-Related Changes in Structural Covariance Networks of Human Anterior and Posterior Hippocampus}

\author{
Xinwei $\mathrm{Li}^{1,2}$, Qiongling $\mathrm{Li}^{1,2}$, Xuetong Wang ${ }^{1,2}$, Deyu $\mathrm{Li}^{1,2 *}$ and Shuyu $\mathrm{Li}^{1,2 *}$ \\ ${ }^{1}$ School of Biological Science and Medical Engineering, Beihang University, Beijing, China, ${ }^{2}$ Beijing Advanced Innovation \\ Centre for Biomedical Engineering, Beihang University, Beijing, China
}

The hippocampus plays an important role in memory function relying on information interaction between distributed brain areas. The hippocampus can be divided into the anterior and posterior sections with different structure and function along its long axis. The aim of this study is to investigate the effects of normal aging on the structural covariance of the anterior hippocampus (aHPC) and the posterior hippocampus (pHPC). In this study, 240 healthy subjects aged 18-89 years were selected and subdivided into young (18-23 years), middle-aged (30-58 years), and older (61-89 years) groups. The aHPC and pHPC was divided based on the location of uncal apex in the MNI space. Then, the structural covariance networks were constructed by examining their covariance in gray matter volumes with other brain regions. Finally, the influence of age on the structural covariance of these hippocampal sections was explored. We found that the aHPC and pHPC had different structural covariance patterns, but both of them were associated with the medial temporal lobe and insula. Moreover, both increased and decreased covariances were found with the aHPC but only increased covariance was found with the pHPC with age ( $p<0.05$, family-wise error corrected). These decreased connections occurred within the default mode network, while the increased connectivity mainly occurred in other memory systems that differ from the hippocampus. This study reveals different age-related influence on the structural networks of the aHPC and pHPC, providing an essential insight into the mechanisms of the hippocampus in normal aging.

Keywords: network, structural covariance, normal aging, anterior hippocampus, posterior hippocampus, MRI

\section{INTRODUCTION}

With the population aging, understanding normal brain changes are as important as understanding demented diseases. Memory decline is a typical characteristic of normal aging. The hippocampus is considered critical in human memory and spatial navigation (Scoville and Milner, 1957; Buzsáki and Moser, 2013). Evidence suggests that hippocampal volume changes throughout the lifespan, which stays relatively stable until the age of 60 shows a sharp decline (Raz et al., 2010; Schuff et al., 2012; Fjell et al., 2013). Functional imaging studies have revealed and hypometabolism (de Leon et al., 2001; Wu et al., 2008) of the hippocampus in aging. Moreover, a reduced fractal dimension of hippocampal dynamics with age was reported (Goldberger et al., 2002; Wink et al., 2006). 
The hippocampus differs in structure and function along its longitudinal axis (Poppenk et al., 2013). The anterior hippocampus (aHPC) and posterior hippocampus (pHPC) vary in pyramidal cell density (Babb et al., 1984; King et al., 2008) and have different developmental trajectories (DeMaster et al., 2014). Compared with young adults, both the aHPC and the pHPC showed volumetric atrophy in old adults (Pruessner et al., 2001; Chen et al., 2010; Rajah et al., 2010), and their rates of atrophy were different (Malykhin et al., 2008; Chen et al., 2010). Besides, an fMRI study reported the functional connectivity of the aHPC and pHPC were differentially affected in aging (Damoiseaux et al., 2016).

For structural connectivity, the structural covariance network (SCN) approach provides an effective way to characterize interregional structural covariance pattern of gray matter (GM) morphological properties (Mechelli et al., 2005; Modinos et al., 2009; Seeley et al., 2009; Zielinski et al., 2010; Montembeault et al., 2012; Li et al., 2013; DuPre and Spreng, 2017). The GM morphological covariance may result from direct white matter connection or neuronal co-activation (Alexander-Bloch et al., 2013). Studies have revealed a consistency among SCNs, anatomical connectivity networks, and functional connectivity networks, which provides strong support for using SCN mapping approach to assess network integrity. Age-related alteration of structural covariance in sensorimotor and cognitive networks has been found (Montembeault et al., 2012; Li et al., 2013). However, the effects of aging on the structural covariance of the aHPC and $\mathrm{pHPC}$ remain to be studied, which may provide insights into the hippocampal-related mechanism of aging and demented diseases.

In this study, we utilized a seed-based SCN approach to investigate the anterior and posterior hippocampal structural networks in 240 healthy subjects that were subdivided into young, middle-aged, and elderly groups. We first defined aHPC and pHPC based on the location of uncal apex in the MNI space. Then, we identified the SCNs seeding from aHPC and pHPC and compared the structural covariance differences between age groups. We expected the SCNs of the aHPC and pHPC have different patterns and were differently affected by age.

\section{MATERIALS AND METHODS}

\section{Participants}

The MRI data were obtained from the publicly available Open Access Series of Imaging Studies (OASIS) database (Marcus et al., 2007). The OASIS database consists of 416 subjects aged 18-96, including 100 mild dementia and 316 healthy subjects. Based on the age distribution of the OASIS database, we selected 240 participants from the healthy subcohort and grouped them into young (18-23 years), middle-aged (30-58 years), and elderly (6189 years) groups, with 80 participants in each group (see Table 1). All the subjects are right-handed and cognitively normal, with the Mini-Mental State Examination scores (Folstein et al., 1975) above 29 and the Clinical Dementia Rating scores (Folstein et al., 1975) equal zero. The same group of subjects was used in our previous study (Li et al., 2013).

\section{Data Acquisition}

All MRI scans were performed on 1.5 Tesla Siemens scanners. For each individual, three to four T1-weighted images were acquired using a magnetization-prepared rapid gradient echo (MPRAGE) sequence with the following parameters: repetition time $=9.7 \mathrm{~ms}$; echo time $=4 \mathrm{~ms}$; inversion time $=20 \mathrm{~ms}$; delay time $=200 \mathrm{~ms}$; flip angle $=10^{\circ}$; matrix $=256 \times 256$; field of view $=256 \mathrm{~mm}$; slices $=128$; slice thickness $=1.25 \mathrm{~mm}$. After motion corrected, the images of each subject were averaged to improve the contrastto-noise ratio.

\section{Image Preprocessing}

We used the VBM8 toolbox ${ }^{1}$ runs within SPM8 to implement voxel-based morphometry analysis (Ashburner and Friston, 2000) of the structural images. The acquired anatomical images were tissue classified into GM, white matter and cerebrospinal fluid images using tissue priors. Then, the segmented images were bias corrected and registered to a standard space using an affine transformation and a high-dimensional non-linear registration approach (Ashburner and Friston, 2005). Next, modulation of the segmented images was performed to correct for different individual brain size by using the non-linear registration parameters. Finally, the modulated GM segments were smoothed using an isotropic $12 \mathrm{~mm}$ full-width at half maximum Gaussian kernel for the structural covariance analysis.

\section{Definition of the Hippocampal Seeds}

Following previous studies (Poppenk et al., 2013; Persson et al., 2014), we adopted a MNI-coordinate-based segmentation method to partition the hippocampus. The hippocampus was identified using the Harvard-Oxford subcortical structural atlas (Desikan et al., 2006) from the FSL Software Library (Smith et al., 2004). Next, the left and right hippocampi were divided into the anterior and posterior sections separately based on the location of uncal apex in the MNI space (i.e., $Y=-21 \mathrm{~mm}$ ) (Poppenk et al., 2013). To avoid contamination effects between the aHPC and the pHPC, we removed a $2-\mathrm{mm}$ coronal slice from each of the two adjacent ends (see Figure 1). For each subject, we measured the mean volumes of the hippocampal subfields from the modulated GM images using the MarsBar ROI toolbox ${ }^{2}$. Then a quadratic regression model was used to investigate age effects on the mean volumes of the anterior and posterior hippocampal segments. We also assessed the age-related hippocampal volumetric dispersion. To do so, for age $=t$, we calculated the variance of hippocampal volumes of subjects with age $\in[t-2, t+2]$ and examined its quadratic relationship with age.

\footnotetext{
${ }^{1}$ http://www.neuro.uni-jena.de/vbm/

${ }^{2}$ http://marsbar.sourceforge.net/
}

TABLE 1 | Participant characteristics by age group.

\begin{tabular}{lcc}
\hline Group & Sample size (Females) & Age in years (mean \pm SD) \\
\hline Young & $80(50)$ & $18-23(20.66 \pm 1.47)$ \\
Middle-aged & $80(50)$ & $30-58(47.43 \pm 8.23)$ \\
Old & $80(55)$ & $61-89(73.75 \pm 7.12)$
\end{tabular}




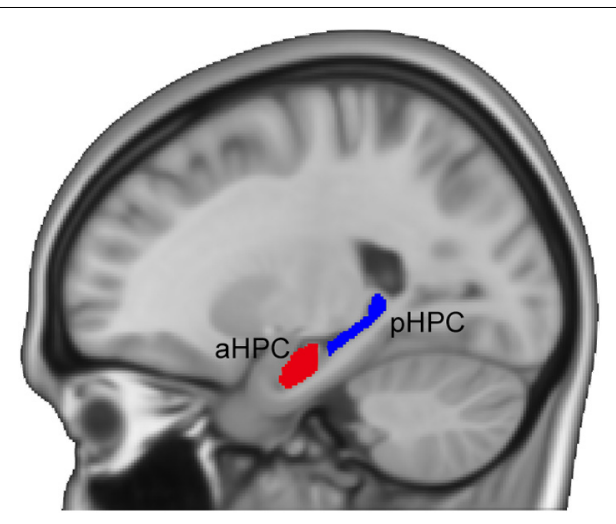

FIGURE 1 | Illustration of the anterior and posterior hippocampal seeds. aHPC, anterior hippocampus; pHPC, posterior hippocampus.

\section{Structural Covariance Analysis}

Four separate regression analyses were executed on the modulated GM images data to map SCNs of the bilateral aHPC and $\mathrm{pHPC}$ in the young group. The model fitted the target voxel GM volume $Y$ as:

$$
Y \sim \beta_{0}+\beta_{1}(\text { Seed })+\beta_{2}(\text { Gender })
$$

where $\beta_{0}$ is the intercept term, $\beta_{1}$ model the relationship between the target voxel volume and the seed volume, and the Gender term was included as a nuisance variable. Total intracranial volume was not included because the modulation step already considered the brain size differences. These statistical analyses enable us to determine voxels that expressed a significant positive correlation with each seed. The criterion for significance was set at height and extent thresholds of $p<0.05$, family-wise error (FWE) corrected for multiple comparisons. The resulting correlation maps were displayed on a standard brain template using the BrainNet Viewer (Xia et al., 2013) to allow qualitative comparisons the structural covariance patterns of hippocampal seeds.

We further assessed the influence of age on the regional structural covariance between the hippocampus and the rest brain regions by using a classic linear interaction model (Lerch et al., 2006). For any two age groups, the target voxel volume $Y$ was modeled as follows:

$$
\begin{aligned}
\mathrm{Y} \sim \beta_{0}+\beta_{1}(\text { Seed })+\beta_{2}(\text { Group })+ & \beta_{3}(\text { Gender })+ \\
& \beta_{4}(\text { Group } \times \text { Seed })
\end{aligned}
$$

where $\beta_{0}$ is the intercept term, $\beta_{1} \sim \beta_{4}$ models the relationship between the target voxel volume and the seed volume, group term, gender term, and interaction term (group by seed), respectively. To obtain between-group differences, specific $t$ contrasts were established to test the statistical significance of the interaction term. Clusters with height and extent thresholds set at $p<0.05$ (FWE corrected) were considered significant.

\section{RESULTS}

\section{Hippocampal Volume Analyses}

Results for the regression analysis of anterior and posterior hippocampal mean GM volumes versus age are presented in Figure 2. Similar nonlinear relationship between the bilateral hippocampal volumes and age were found: the volumes slightly increased before the age of 50 and then decreased sharply (left aHPC: $R^{2}=0.187, p<0.001$; right aHPC: $R^{2}=0.136, p<0.001$; left pHPC: $R^{2}=0.089, p<0.001$; right pHPC: $R^{2}=0.106$, $p<0.001)$. Moreover, the results suggested that the mean volume of the aHPC was larger than the pHPC, and the left hippocampal volume was slightly greater than the right side. In addition, we found the variance of the bilateral anterior hippocampal volumes has an age-related U-shaped relationship (left aHPC: $R^{2}=0.489$, $p<0.001$; right aHPC: $\left.R^{2}=0.666, p<0.001\right)$. Specifically, the anterior hippocampal volumes of the young and old subjects were more dispersed than the middle age. However, the variance of the posterior hippocampal volumes did not significantly relate to age (left pHPC: $R^{2}=0.015, p=0.646$; right pHPC: $R^{2}=0.051$, $p=0.215)$.

\section{Structural Covariance Networks of the Anterior and Posterior Hippocampus}

The SCNs seeding from the aHPC and pHPC in the young participants are presented in Figure 3 ( $p<0.05$, FWE corrected). The aHPC correlated with the bilateral temporal lobe (including the superior, middle and inferior temporal, parahippocampal gryi, entorhinal cortex, fusiform and temporal pole), amygdalae, insula and posterior cingulate gyrus, orbitofrontal cortex, as well as left superior frontal gyrus. For the pHPC, its covariance maps involved the bilateral medial temporal regions (including the parahippocampal gyrus, entorhinal cortex and fusiform), amygdalae and insula. Noted that the regions correlated with both the aHPC and pHPC were mainly located in the medial temporal lobe and insula.

\section{Age-Related Differences Within the Anterior Hippocampal Network}

Within the anterior hippocampal network, significant betweengroup differences were only observed between the young group and the old group $(p<0.05$, FWE corrected, Figure 4 and Table 2). Specifically, the left and right aHPC showed decreased positive correlation with the ipsilateral parahippocampus and increased positive correlation with the ipsilateral amygdala in the old group relative to the young group. Moreover, compared to the young group, the left aHPC exhibited lower structural covariance with the left precuneus and greater structural covariance with the right putamen in the old group.

\section{Age-Related Differences Within the Posterior Hippocampal Network}

Within the posterior hippocampal network, only increased structural associations were found in the old group relative to younger adults (mainly the young group, $p<0.05$, FWE corrected, see Figure 5 and Table 2). For the left pHPC, the 

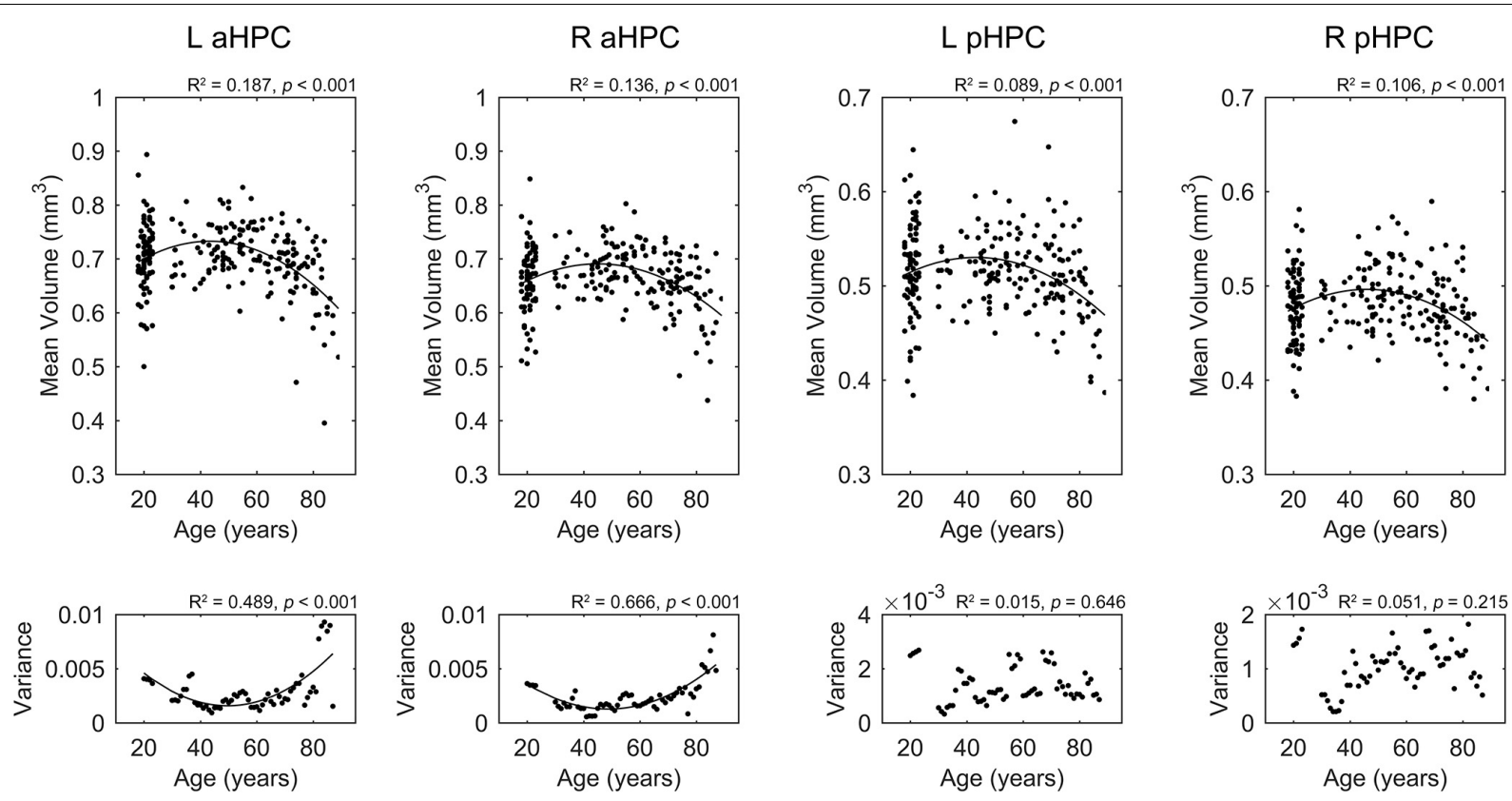

FIGURE 2 | Life-span trajectories of the anterior and posterior hippocampal mean gray matter volumes. The lower row shows the relationship between age and the variance of hippocampal volumes in a small age range. aHPC, anterior hippocampus; pHPC, posterior hippocampus; L, left, R, right.

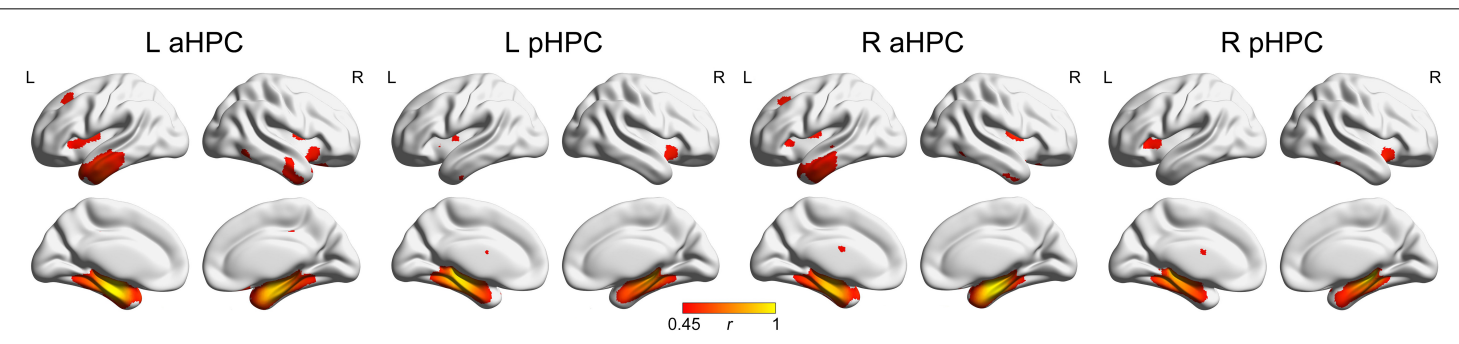

FIGURE 3 | Structural covariance networks of the anterior and posterior hippocampus in the Young group. Regions with $P_{\mathrm{FWE}}<0.05$ are presented as correlation coefficient values. aHPC, anterior hippocampus; pHPC, posterior hippocampus; L, left; R, right.

old group exhibited significantly increased connectivity with the right caudate related to the young group. For the right $\mathrm{pHPC}$, its connection with bilateral putamen was negative in the young group but was positive in the old group. Similarly, the right pHPC and temporal pole was negatively related in the middle-aged group but positively related in the old group.

\section{DISCUSSION}

Here, we studied the age-related structural covariance alterations of the aHPC and pHPC using a seed-based SCN approach. We found that the SCNs seeding from the aHPC and pHPC in the young adults were different from each other, but both of them related with the medial temporal lobe and insula. In addition, the structural covariance differences within the anterior hippocampal network were mainly between the young group and the old group with both decreased and increased positive structural associations. While compared to the younger adults, only increased structural associations were found in the old group within the posterior hippocampal network.

We observed that the volumes of aHPC/pHPC slightly increased from young to middle age, and then decreased sharply with age. In line with this finding, several morphometric studies reported an inverted $U$ pattern of the hippocampal volume changes with age (Walhovd et al., 2005; Li et al., 2014). As the hippocampus is important in memory processing, this pattern may partially explain the similar age-related memory change trajectory (Nyberg et al., 2012). Interestingly, we found that the anterior hippocampal volumes of the young and old subjects are more dispersed than the middle age, may pointing to stronger heterogeneity memory ability in young and old subjects. Whether this age-related dispersion due to the sample selection or other reasons requires further analysis.

Structural covariance analyses suggested that the aHPC connected with temporal lobe, amygdala, insula, and orbitofrontal cortex ( $p<0.05$, FWE corrected), which agree with 

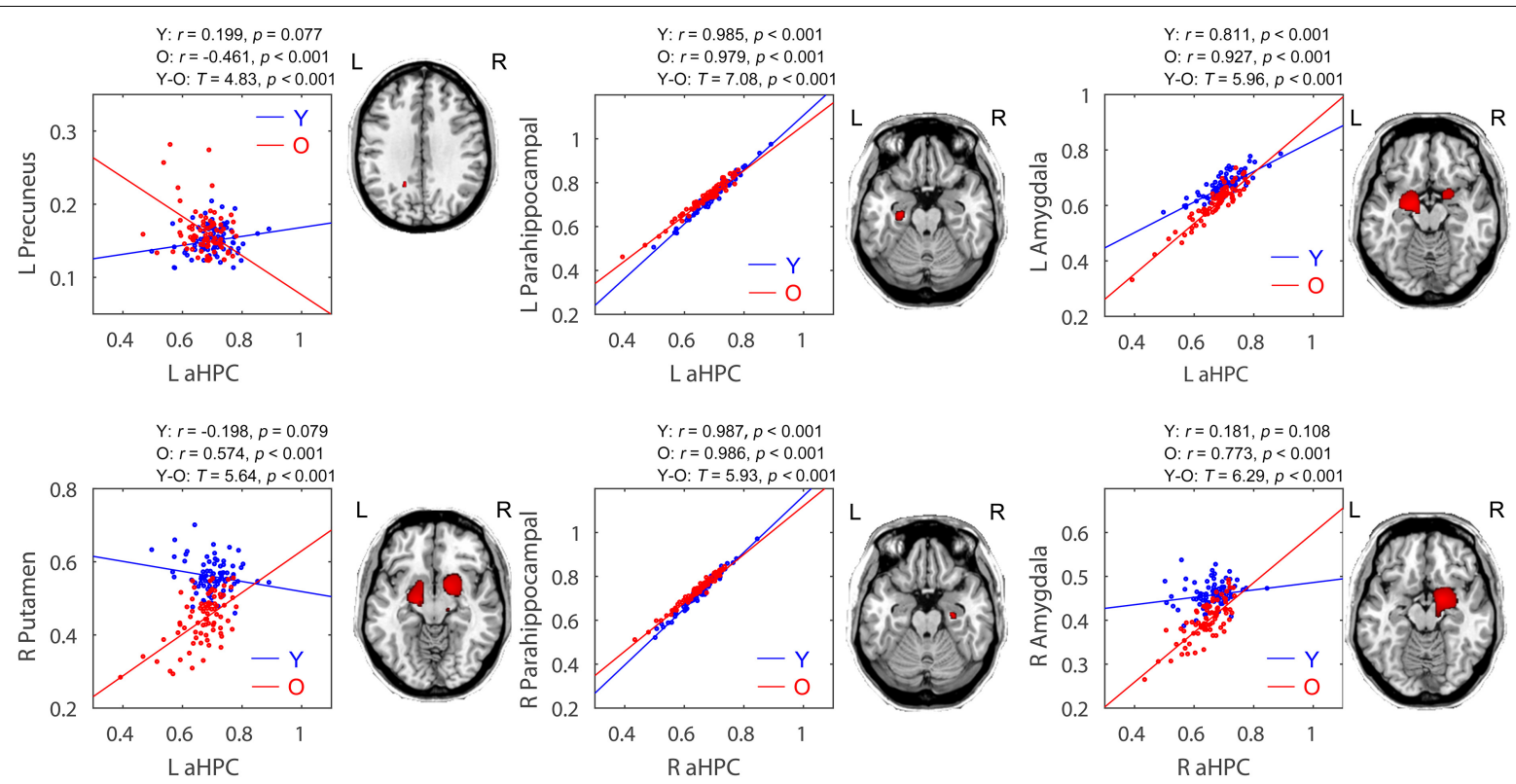

FIGURE 4 | Age-related group differences in structural covariance of the anterior hippocampus. Correlations between the mean volume of the anterior hippocampus and the regional gray matter volumes extracted from a 4-mm-radius sphere centered on the peak voxel of a significant cluster ( $P_{\mathrm{FWE}}<0.05$, shown on the right) are displayed. Y, young group; O, old group; L, left; R, right.

TABLE 2 | Significant between-group differences in structural association between hippocampal seeds and other anatomical regions.

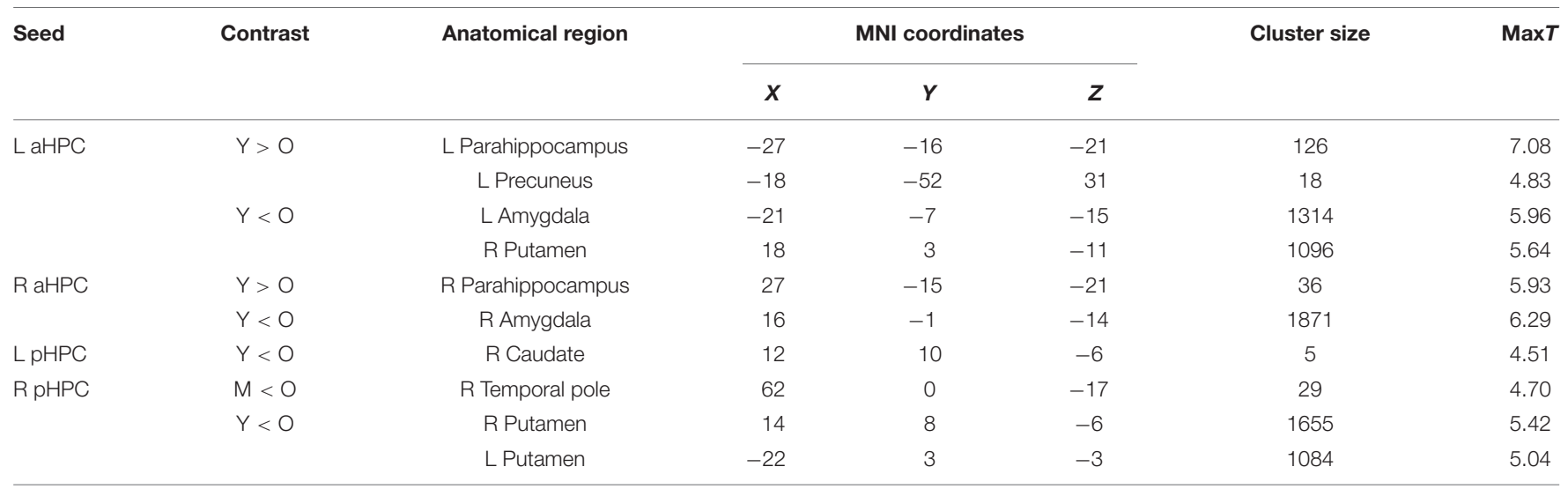

P< 0.05, FWE corrected. Abbreviations: L, left; R, right; aHPC, anterior hippocampus; $p H P C$, posterior hippocampus; $Y$, young group; M, middle-aged group; O, old group.

previous studies (Kier et al., 2004; Smith et al., 2009; Catenoix et al., 2011). And the pHPC was covariant with medial temporal amygdala, and insula ( $p<0.05$, FWE corrected) showing consistent connections with previous studies by using fMRI and tractography (Kahn et al., 2008; Poppenk and Moscovitch, 2011; Poppenk et al., 2013). The common related regions with both aHPC and pHPC were mainly located in the medial temporal lobe where the hippocampus located.

Age-related decrements in structural covariance were observed in the aHPC-related SCNs ( $p<0.05$, FWE corrected). In particular, the parahippocampal gyrus and precuneus showed reduced association with the aHPC seed in old adults relative to young adults. The parahippocampal gyrus is considered as a mediator between the cortical DMN subsystem and the hippocampus (Ward et al., 2014), and the integrity of the cortico-parahippocampus-hippocampus circuit is important for learning and episodic memory (Witter et al., 2000; Van Strien et al., 2009). Therefore, the weakened parahippocampushippocampus connection may lead to memory deficits in normal elderly, and result in decreased structural covariance between the hippocampus and cortical regions, such as the precuneus found in this study. Besides, the decreased connectivity between the precuneus and hippocampus might result from very early betaamyloid deposition of the precuneus in elderly subjects (Sheline et al., 2010). The abnormal synaptic activity caused by amyloid deposition might disrupt cortico-hippocampal connectivity, which then results in hippocampal atrophy (Mormino et al., 2009). 

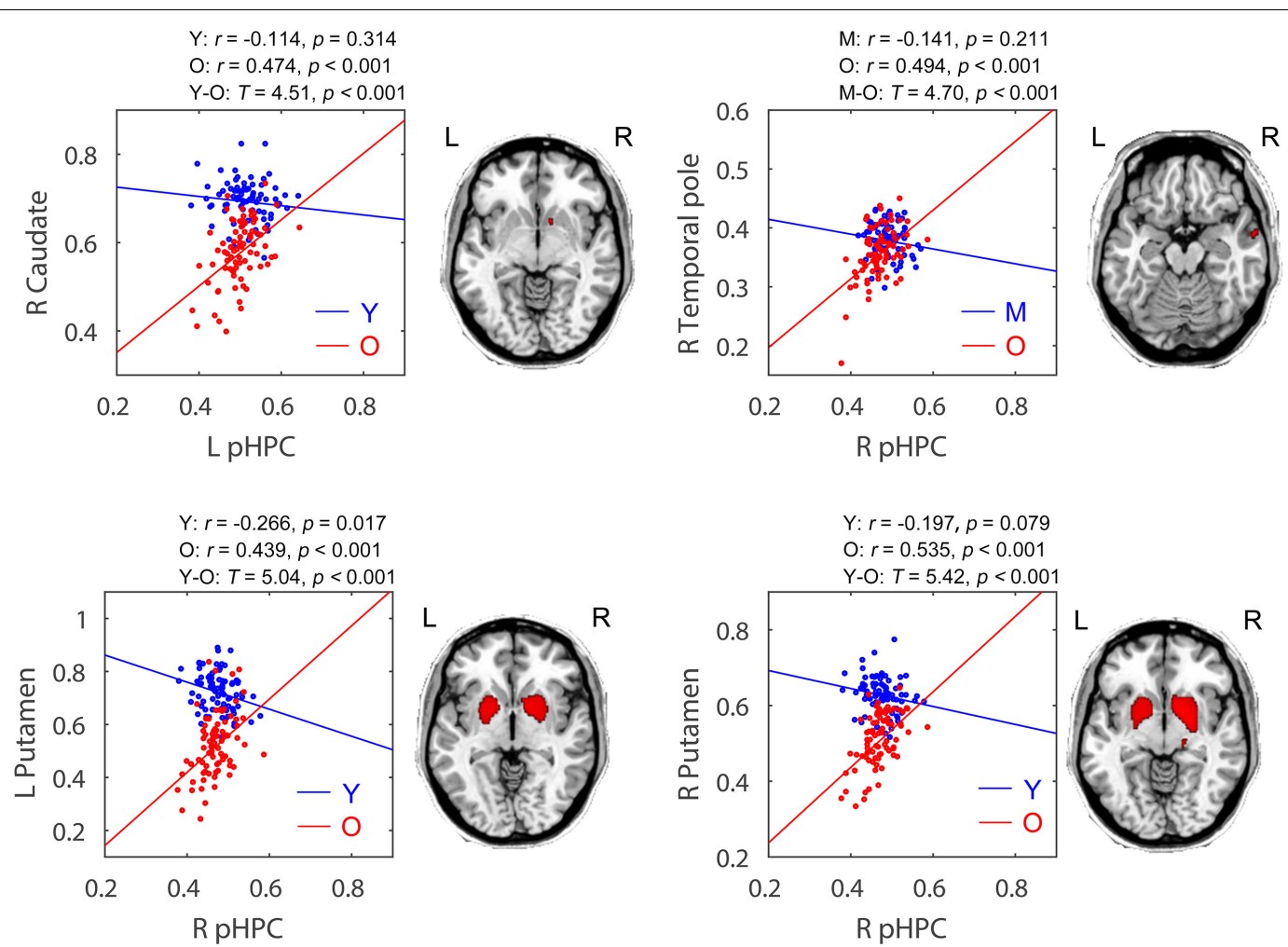

FIGURE 5 | Age-related group differences in structural covariance of the posterior hippocampus. Correlations between the mean volume of the posterior hippocampus and the regional gray matter volumes extracted from a 4-mm-radius sphere centered on the peak voxel of a significant cluster $\left(P_{\mathrm{FWE}}<0.05\right.$, shown on the right) are displayed. aHPC, anterior hippocampus; pHPC, posterior hippocampus; Y, young group; M, middle-aged group; O, old group; L, left; R, right.

Note that the parahippocampal gyrus, precuneus, and hippocampus are all components of DMN (Andrews-Hanna et al., 2014). Thus, our findings may indicate that aging is associated with decreased structural covariance within the DMN, which is in keeping with observations from previous SCN studies (Montembeault et al., 2012; Li et al., 2013; Spreng and Turner, 2013). A previous study reported decreased fractal complexity in DMN with age using multifractal analysis of fMRI series (Ni et al., 2014). Moreover, aging-related decrements in functional connectivity (Damoiseaux et al., 2008; Tomasi and Volkow, 2012) and white matter integrity (Damoiseaux et al., 2009; Brown et al., 2015) of DMN were also reported. Since $\mathrm{DMN}$ is known to play a role in episodic memory processing (Greicius et al., 2004, 2009), its decreased integrity could underlie memory impairment in senior populations (Salami et al., 2014).

Additionally, our data suggest that the influence of age on the structural connectivity between the hippocampus and cortical DMN nodes may be limited to the anterior portion of the hippocampus. Similarly, Salami et al. (2014) revealed reduced functional connectivity between the cortical DMN subsystems and more anteriorly located hippocampus with advancing age. Several fMRI studies have demonstrated the aHPC as part of DMN was engaged in episodic memory (autobiographical memory) processing (Zeidman and Maguire, 2016). However, some studies found no age-related differences for the connectivity between the aHPC and DMN regions (Koch et al., 2010; Damoiseaux et al., 2016), while others reported lower connectivity between the $\mathrm{PHPC}$ and DMN regions in older adults (Andrews-Hanna et al., 2007; Damoiseaux et al., 2016). These discrepancies may be due to methodological differences, notably in the type of measurements and sample characteristics, which should be further investigated.

Moreover, age-related increments in structural covariance were observed in both the aHPC- and pHPC-related SCNs ( $p<0.05$, FWE corrected). Particularly, compared to young adults, the putamen and amygdala showed increased associations within the aHPC-related SCNs in old adults. Within the pHPC-related SCNs, the putamen, caudate, and temporal pole showed increased associations in old adults relative to younger adults. The putamen and caudate form the dorsal striatum. In fact, the hippocampus, dorsal striatum, and amygdala belong to different memory systems and play different roles in information acquisition (McDonald and White, 1993). The dorsal striatum and hippocampus cooperate to support episodic memory function (Sadeh et al., 2011), while the amygdala plays a role in regulating these two memory systems (Packard and Teather, 1998). We speculated that the age-related increment in hippocampal structural covariance may reflect the compensatory mechanism or dedifferentiation effects of the brain memory systems during aging (Dennis and Cabeza, 2011; Oedekoven et al., 2015). 
The greater structural covariance between the hippocampus and dorsal striatum (caudate-putamen) in older adults may also be related to non-optimal dopamine processing. The CA1 area of the hippocampus receives dopaminergic modulation from the ventral tegmental area, which plays a vital role in synaptic plasticity of the hippocampus (Lisman and Grace, 2005). But the ventral tegmental area suffers from dopamine neurons loss (Siddiqi et al., 1999) and reduced dopamine transporter function (Salvatore et al., 2003) with age. However, the dorsal striatum, another area in the dopamine system, increases its dopamine synthesis capacity in aging (Braskie et al., 2008). Thus, the increased connections between the hippocampus and dorsal striatum during aging suggest compensation for deficits in the ventral tegmental area, which may represent non-optimal dopamine system functioning.

The SCN method used in this study provides an effective way to construct brain networks from medical images, which complements the signal analysis methods (Liu et al., 2015). However, since aging is not only characterized by brain deficits but also decline in multiple organ functions, it is interesting to utilize the integrative approaches within the new filed of network physiology to study the effects of aging on brain-brain or brainorgan networks in future (Bashan et al., 2012; Bartsch et al., 2015; Ivanov et al., 2016). In addition, it is worth noting that brain networks have a fractal property of hierarchical modularity, which confers robustness of network function (Bullmore and Sporns, 2012). Future studies using fractal analysis approaches (Meunier et al., 2010; Xue and Bogdan, 2017) to study the

\section{REFERENCES}

Alexander-Bloch, A., Giedd, J. N., and Bullmore, E. (2013). Imaging structural co-variance between human brain regions. Nat. Rev. Neurosci. 14, 322-336. doi: $10.1038 / \mathrm{nrn} 3465$

Andrews-Hanna, J. R., Snyder, A. Z., Vincent, J. L., Lustig, C., Head, D., Raichle, M. E., et al. (2007). Disruption of large-scale brain systems in advanced aging. Neuron 56, 924-935. doi: 10.1016/j.neuron.2007.10.038

Andrews-Hanna, J. R., Smallwood, J., and Spreng, R. N. (2014). The default network and self-generated thought: component processes, dynamic control, and clinical relevance. Ann. N. Y. Acad. Sci. 1316, 29-52. doi: 10.1111/nyas. 12360

Ashburner, J., and Friston, K. J. (2000). Voxel-based morphometry-the methods. Neuroimage 11, 805-821. doi: 10.1006/nimg.2000.0582

Ashburner, J., and Friston, K. J. (2005). Unified segmentation. Neuroimage 26, 839-851. doi: 10.1016/j.neuroimage.2005.02.018

Babb, T. L., Lieb, J. P., Brown, W. J., Pretorius, J., and Crandall, P. H. (1984). Distribution of pyramidal cell density and hyperexcitability in the epileptic human hippocampal formation. Epilepsia 25, 721-728. doi: 10.1111/j.15281157.1984.tb03483.x

Bartsch, R. P., Liu, K. K., Bashan, A., and Ivanov, P. C. (2015). Network physiology: how organ systems dynamically interact. PLoS One 10:e0142143. doi: 10.1371/ journal.pone.0142143

Bashan, A., Bartsch, R. P., Kantelhardt, J. W., Havlin, S., and Ivanov, P. C. (2012). Network physiology reveals relations between network topology and physiological function. Nat. Commun. 3:702. doi: 10.1038/ncomms 1705

Braskie, M. N., Wilcox, C. E., Landau, S. M., O’neil, J. P., Baker, S. L., Madison, C. M., et al. (2008). Relationship of striatal dopamine synthesis capacity to age and cognition. J. Neurosci. 28, 14320-14328. doi: 10.1523/JNEUROSCI.372908.2008 complexity and heterogeneity of hippocampal networks could advance our understanding of the brain in normal aging.

\section{AUTHOR CONTRIBUTIONS}

XL designed and performed the experiments, analyzed the data, and drafted the manuscript. QL and XW helped to analyze the data and to draft the manuscript. DL and SL contributed to the study design, coordination, and final approval of the manuscript. All authors read and approved this version to be published.

\section{FUNDING}

This work was supported by the National Natural Science Foundation of China (Grant Nos. 81622025 and 81471731), the Fundamental Research Funds for the Central Universities (Grant No. YWF-17-BJ-J-11), the Innovation Foundation of BUAA for Ph.D. Students, and Academic Excellence Foundation of BUAA for Ph.D. Students. The OASIS project was supported by the NIH Grant Nos. P50 AG05681, P01 AG03991, R01 AG021910, P50 MH071616, U24 RR021382, and R01 MH56584.

\section{ACKNOWLEDGMENTS}

The authors would like to thank the OASIS project for making the MRI data freely available.

Brown, C. A., Hakun, J. G., Zhu, Z. D., Johnson, N. F., and Gold, B. T. (2015). White matter microstructure contributes to age-related declines in taskinduced deactivation of the default mode network. Front. Aging Neurosci. 7:194. doi: 10.3389/fnagi.2015.00194

Bullmore, E., and Sporns, O. (2012). The economy of brain network organization. Nat. Rev. Neurosci. 13, 336-349. doi: 10.1038/nrn3214

Buzsáki, G., and Moser, E. I. (2013). Memory, navigation and theta rhythm in the hippocampal-entorhinal system. Nat. Neurosci. 16, 130-138. doi: 10.1038/nn. 3304

Catenoix, H., Magnin, M., Mauguiere, F., and Ryvlin, P. (2011). Evoked potential study of hippocampal efferent projections in the human brain. Clin. Neurophysiol. 122, 2488-2497. doi: 10.1016/j.clinph.2011.05.007

Chen, K. H., Chuah, L. Y., Sim, S. K., and Chee, M. W. (2010). Hippocampal regionspecific contributions to memory performance in normal elderly. Brain Cogn. 72, 400-407. doi: 10.1016/j.bandc.2009.11.007

Damoiseaux, J. S., Beckmann, C. F., Arigita, E. J., Barkhof, F., Scheltens, P., Stam, C. J., et al. (2008). Reduced resting-state brain activity in the "default network" in normal aging. Cereb. Cortex 18, 1856-1864. doi: 10.1093/cercor/bhm207

Damoiseaux, J. S., Smith, S. M., Witter, M. P., Sanz-Arigita, E. J., Barkhof, F., Scheltens, P., et al. (2009). White matter tract integrity in aging and Alzheimer's disease. Hum. Brain Mapp. 30, 1051-1059. doi: 10.1002/hbm.20563

Damoiseaux, J. S., Viviano, R. P., Yuan, P., and Raz, N. (2016). Differential effect of age on posterior and anterior hippocampal functional connectivity. Neuroimage 133, 468-476. doi: 10.1016/j.neuroimage.2016.03.047

de Leon, M. J., Convit, A., Wolf, O. T., Tarshish, C. T., DeSanti, S., Rusinek, H., et al. (2001). Prediction of cognitive decline in normal elderly subjects with 2-[18F] fluoro-2-deoxy-D-glucose/positron-emission tomography (FDG/PET). Proc. Natl. Acad. Sci. U.S.A. 98, 10966-10971. doi: 10.1073/pnas.191 044198

DeMaster, D., Pathman, T., Lee, J. K., and Ghetti, S. (2014). Structural Development of the Hippocampus and Episodic Memory: Developmental Differences Along 
the Anterior/Posterior Axis. Cereb. Cortex 24, 3036-3045. doi: 10.1093/cercor/ bht160

Dennis, N. A., and Cabeza, R. (2011). Age-related dedifferentiation of learning systems: an fMRI study of implicit and explicit learning. Neurobiol. Aging 32, 2318.e17-30. doi: 10.1016/j.neurobiolaging.2010.04.004

Desikan, R. S., Ségonne, F., Fischl, B., Quinn, B. T., Dickerson, B. C., Blacker, D., et al. (2006). An automated labeling system for subdividing the human cerebral cortex on MRI scans into gyral based regions of interest. Neuroimage 31, 968-980. doi: 10.1016/j.neuroimage.2006.01.021

DuPre, E., and Spreng, R. N. (2017). Structural covariance networks across the lifespan, from 6-94 years of age. Netw. Neurosci. 1, 302-323.

Fjell, A. M., Westlye, L. T., Grydeland, H., Amlien, I., Espeseth, T., Reinvang, I., et al. (2013). Critical ages in the life course of the adult brain: nonlinear subcortical aging. Neurobiol. Aging 34, 2239-2247. doi: 10.1016/j. neurobiolaging.2013.04.006

Folstein, M. F., Folstein, S. E., and Mchugh, P. R. (1975). "Mini-mental state": a practical method for grading the cognitive state of patients for the clinician. J. Psychiatr. Res. 12, 189-198. doi: 10.1016/0022-3956(75)90026-6

Goldberger, A. L., Amaral, L. A., Hausdorff, J. M., Ivanov, P. C., Peng, C.-K., and Stanley, H. E. (2002). Fractal dynamics in physiology: alterations with disease and aging. Proc. Natl. Acad. Sci. U.S.A. 99, 2466-2472. doi: 10.1073/ pnas.012579499

Greicius, M. D., Srivastava, G., Reiss, A. L., and Menon, V. (2004). Defaultmode network activity distinguishes Alzheimer's disease from healthy aging: evidence from functional MRI. Proc. Natl. Acad. Sci. U.S.A. 101, 4637-4642. doi: 10.1073/pnas.0308627101

Greicius, M. D., Supekar, K., Menon, V., and Dougherty, R. F. (2009). Restingstate functional connectivity reflects structural connectivity in the default mode network. Cereb. Cortex 19, 72-78. doi: 10.1093/cercor/bhn059

Ivanov, P. C., Liu, K. K., and Bartsch, R. P. (2016). Focus on the emerging new fields of network physiology and network medicine. N. J. Phys. 18:100201. doi: 10.1111 /ede. 12187

Kahn, I., Andrews-Hanna, J. R., Vincent, J. L., Snyder, A. Z., and Buckner, R. L. (2008). Distinct cortical anatomy linked to subregions of the medial temporal lobe revealed by intrinsic functional connectivity. J. Neurophysiol. 100, 129-139. doi: 10.1152/jn.00077.2008

Kier, E. L., Staib, L. H., Davis, L. M., and Bronen, R. A. (2004). MR imaging of the temporal stem: anatomic dissection tractography of the uncinate fasciculus, inferior occipitofrontal fasciculus, and Meyer's loop of the optic radiation. Am. J. Neuroradiol. 25, 677-691.

King, K. G., Glodzik, L., Liu, S., Babb, J. S., De Leon, M. J., and Gonen, O. (2008). Anteroposterior hippocampal metabolic heterogeneity: three-dimensional multivoxel proton $1 \mathrm{H}$ MR spectroscopic imaging-initial findings. Radiology 249, 242-250. doi: 10.1148/radiol.2491071500

Koch, W., Teipel, S., Mueller, S., Buerger, K., Bokde, A. L., Hampel, H., et al. (2010). Effects of aging on default mode network activity in resting state fMRI: does the method of analysis matter? Neuroimage 51, 280-287. doi: 10.1016/j. neuroimage.2009.12.008

Lerch, J. P., Worsley, K., Shaw, W. P., Greenstein, D. K., Lenroot, R. K., Giedd, J., et al. (2006). Mapping anatomical correlations across cerebral cortex (MACACC) using cortical thickness from MRI. Neuroimage 31, 993-1003. doi: 10.1016/j.neuroimage.2006.01.042

Li, W., Wu, B., Batrachenko, A., Bancroft-Wu, V., Morey, R. A., Shashi, V., et al. (2014). Differential developmental trajectories of magnetic susceptibility in human brain gray and white matter over the lifespan. Hum. Brain Mapp. 35, 2698-2713. doi: 10.1002/hbm.22360

Li, X., Pu, F., Fan, Y., Niu, H., Li, S., and Li, D. (2013). Age-related changes in brain structural covariance networks. Front. Hum. Neurosci. 7:98. doi: 10.3389/ fnhum.2013.00098

Lisman, J. E., and Grace, A. A. (2005). The hippocampal-VTA loop: controlling the entry of information into long-term memory. Neuron 46, 703-713. doi: 10.1016/j.neuron.2005.05.002

Liu, K. K., Bartsch, R. P., Lin, A., Mantegna, R. N., and Ivanov, P. C. (2015). Plasticity of brain wave network interactions and evolution across physiologic states. Front. Neural Circ. 9:62. doi: 10.3389/fncir.2015.00062

Malykhin, N. V., Bouchard, T. P., Camicioli, R., and Coupland, N. J. (2008). Aging hippocampus and amygdala. Neuroreport 19, 543-547. doi: 10.1097/ WNR.0b013e3282f8b18c
Marcus, D. S., Wang, T. H., Parker, J., Csernansky, J. G., Morris, J. C., and Buckner, R. L. (2007). Open Access Series of Imaging Studies (OASIS): cross-sectional MRI data in young, middle aged, nondemented, and demented older adults. J. Cogn. Neurosci. 19, 1498-1507. doi: 10.1162/jocn.2007.19.9.1498

McDonald, R. J., and White, N. M. (1993). A triple dissociation of memory systems: hippocampus, amygdala, and dorsal striatum. Behav. Neurosci. 107, 3-22. doi: 10.1037/0735-7044.107.1.3

Mechelli, A., Friston, K. J., Frackowiak, R. S., and Price, C. J. (2005). Structural covariance in the human cortex. J. Neurosci. 25, 8303-8310. doi: 10.1523/ JNEUROSCI.0357-05.2005

Meunier, D., Lambiotte, R., and Bullmore, E. T. (2010). Modular and hierarchically modular organization of brain networks. Front. Neuroscience 4:200. doi: 10.3389/fnins.2010.00200

Modinos, G., Vercammen, A., Mechelli, A., Knegtering, H., Mcguire, P. K., and Aleman, A. (2009). Structural covariance in the hallucinating brain: a voxelbased morphometry study. J. Psychiatry Neurosci. 34, 465-469.

Montembeault, M., Joubert, S., Doyon, J., Carrier, J., Gagnon, J. F., Monchi, O., et al. (2012). The impact of aging on gray matter structural covariance networks. Neuroimage 63, 754-759. doi: 10.1016/j.neuroimage.2012.06.052

Mormino, E., Kluth, J., Madison, C., Rabinovici, G., Baker, S., Miller, B., et al. (2009). Episodic memory loss is related to hippocampal-mediated $\beta$-amyloid deposition in elderly subjects. Brain 132, 1310-1323. doi: 10.1093/brain/awn320

Ni, H., Huang, X., Ning, X., Huo, C., Liu, T., and Ben, D. (2014). Multifractal analysis of resting state fMRI series in default mode network: age and gender effects. Chin. Sci. Bull. 59, 3107-3113. doi: 10.1007/s11434-014-0355-x

Nyberg, L., Lövdén, M., Riklund, K., Lindenberger, U., and Bäckman, L. (2012). Memory aging and brain maintenance. Trends Cogn. Sci. 16, 292-305. doi: 10.1016/j.tics.2012.04.005

Oedekoven, C. S., Jansen, A., Keidel, J. L., Kircher, T., and Leube, D. (2015). The influence of age and mild cognitive impairment on associative memory performance and underlying brain networks. Brain Imaging Behav. 9, 776-789. doi: $10.1007 / \mathrm{s} 11682-014-9335-7$

Packard, M. G., and Teather, L. A. (1998). Amygdala modulation of multiple memory systems: hippocampus and caudate-putamen. Neurobiol. Learn. Mem. 69, 163-203. doi: 10.1006/nlme.1997.3815

Persson, J., Spreng, R. N., Turner, G., Herlitz, A., Morell, A., Stening, E., et al. (2014). Sex differences in volume and structural covariance of the anterior and posterior hippocampus. Neuroimage 99, 215-225. doi: 10.1016/j.neuroimage. 2014.05.038

Poppenk, J., Evensmoen, H. R., Moscovitch, M., and Nadel, L. (2013). Longaxis specialization of the human hippocampus. Trends Cogn. Sci. 17, 230-240. doi: 10.1016/j.tics.2013.03.005

Poppenk, J., and Moscovitch, M. (2011). A hippocampal marker of recollection memory ability among healthy young adults: contributions of posterior and anterior segments. Neuron 72, 931-937. doi: 10.1016/j.neuron.2011.10.014

Pruessner, J., Collins, D., Pruessner, M., and Evans, A. (2001). Age and gender predict volume decline in the anterior and posterior hippocampus in early adulthood. J. Neurosci. 21, 194-200. doi: 10.1523/JNEUROSCI.21-01-00194. 2001

Rajah, M. N., Kromas, M., Han, J. E., and Pruessner, J. C. (2010). Group differences in anterior hippocampal volume and in the retrieval of spatial and temporal context memory in healthy young versus older adults. Neuropsychologia 48, 4020-4030. doi: 10.1016/j.neuropsychologia.2010.10.010

Raz, N., Ghisletta, P., Rodrigue, K. M., Kennedy, K. M., and Lindenberger, U. (2010). Trajectories of brain aging in middle-aged and older adults: regional and individual differences. Neuroimage 51, 501-511. doi: 10.1016/j.neuroimage. 2010.03.020

Sadeh, T., Shohamy, D., Levy, D. R., Reggev, N., and Maril, A. (2011). Cooperation between the hippocampus and the striatum during episodic encoding. J. Cogn. Neurosci. 23, 1597-1608. doi: 10.1162/jocn.2010.21549

Salami, A., Pudas, S., and Nyberg, L. (2014). Elevated hippocampal resting-state connectivity underlies deficient neurocognitive function in aging. Proc. Natl. Acad. Sci. U.S.A. 111, 17654-17659. doi: 10.1073/pnas.1410233111

Salvatore, M. F., Apparsundaram, S., and Gerhardt, G. A. (2003). Decreased plasma membrane expression of striatal dopamine transporter in aging. Neurobiol. Aging 24, 1147-1154. doi: 10.1016/S0197-4580(03)00129-5

Schuff, N., Tosun, D., Insel, P. S., Chiang, G. C., Truran, D., Aisen, P. S., et al. (2012). Nonlinear time course of brain volume loss in cognitively normal and 
impaired elders. Neurobiol. Aging 33, 845-855. doi: 10.1016/j.neurobiolaging. 2010.07.012

Scoville, W. B., and Milner, B. (1957). Loss of recent memory after bilateral hippocampal lesions. J. Neurol. Neurosurg. Psychiatry 20, 11-21. doi: 10.1136/ jnnp.20.1.11

Seeley, W. W., Crawford, R. K., Zhou, J., Miller, B. L., and Greicius, M. D. (2009). Neurodegenerative diseases target large-scale human brain networks. Neuron 62, 42-52. doi: 10.1016/j.neuron.2009.03.024

Sheline, Y. I., Raichle, M. E., Snyder, A. Z., Morris, J. C., Head, D., Wang, S., et al. (2010). Amyloid plaques disrupt resting state default mode network connectivity in cognitively normal elderly. Biol. Psychiatry 67, 584-587. doi: 10.1016/j.biopsych.2009.08.024

Siddiqi, Z., Kemper, T. L., and Killiany, R. (1999). Age-related neuronal loss from the substantia nigra-pars compacta and ventral tegmental area of the rhesus monkey. J. Neuropathol. Exp. Neurol. 58, 959-971. doi: 10.1097/00005072199909000-00006

Smith, C. D., Lori, N. F., Akbudak, E., Sorar, E., Gultepe, E., Shimony, J. S., et al. (2009). MRI diffusion tensor tracking of a new amygdalo-fusiform and hippocampo-fusiform pathway system in humans. J. Magn. Reson. Imaging 29, 1248-1261. doi: 10.1002/jmri.21692

Smith, S. M., Jenkinson, M., Woolrich, M. W., Beckmann, C. F., Behrens, T. E., Johansen-Berg, H., et al. (2004). Advances in functional and structural MR image analysis and implementation as FSL. Neuroimage 23, S208-S219. doi: 10.1016/j.neuroimage.2004.07.051

Spreng, R. N., and Turner, G. R. (2013). Structural covariance of the default network in healthy and pathological aging. J. Neurosci. 33, 15226-15234. doi: 10.1523/JNEUROSCI.2261-13.2013

Tomasi, D., and Volkow, N. D. (2012). Aging and functional brain networks. Mol. Psychiatry 17, 471-558. doi: 10.1038/mp.2011.81

Van Strien, N., Cappaert, N., and Witter, M. (2009). The anatomy of memory: an interactive overview of the parahippocampal-hippocampal network. Nat. Rev. Neurosci. 10, 272-282. doi: 10.1038/nrn2614

Walhovd, K. B., Fjell, A. M., Reinvang, I., Lundervold, A., Dale, A. M., Eilertsen, D. E., et al. (2005). Effects of age on volumes of cortex, white matter and subcortical structures. Neurobiol. Aging 26, 1261-1270. doi: 10.1016/j. neurobiolaging.2005.05.020

Ward, A. M., Schultz, A. P., Huijbers, W., Van Dijk, K. R., Hedden, T., and Sperling, R. A. (2014). The parahippocampal gyrus links the default-mode cortical network with the medial temporal lobe memory system. Hum. Brain Mapp. 35, 1061-1073. doi: 10.1002/hbm.22234

Wink, A. M., Bernard, F., Salvador, R., Bullmore, E., and Suckling, J. (2006). Age and cholinergic effects on hemodynamics and functional coherence of human hippocampus. Neurobiol. Aging 27, 1395-1404. doi: 10.1016/j.neurobiolaging. 2005.08.011

Witter, M. P., Naber, P. A., Van Haeften, T., Machielsen, W. C., Rombouts, S. A., Barkhof, F., et al. (2000). Cortico-hippocampal communication by way of parallel parahippocampal-subicular pathways. Hippocampus 10, 398-410. doi: 10.1002/1098-1063(2000)10:4<398::AID-HIPO6>3.0.CO;2-K

Wu, W., Brickman, A. M., Luchsinger, J., Ferrazzano, P., Pichiule, P., Yoshita, M., et al. (2008). The brain in the age of old: the hippocampal formation is targeted differentially by diseases of late life. Ann. Neurol. 64, 698-706. doi: 10.1002/ana. 21557

Xia, M., Wang, J., and He, Y. (2013). BrainNet viewer: a network visualization tool for human brain connectomics. PLoS One 8:e68910. doi: 10.1371/journal.pone. 0068910

Xue, Y., and Bogdan, P. (2017). Reliable multi-fractal characterization of weighted complex networks: algorithms and implications. Sci. Rep. 7:7487. doi: 10.1038/ s41598-017-07209-5

Zeidman, P., and Maguire, E. A. (2016). Anterior hippocampus: the anatomy of perception, imagination and episodic memory. Nat. Rev. Neurosci. 17, 173-182. doi: 10.1038/nrn.2015.24

Zielinski, B. A., Gennatas, E. D., Zhou, J., and Seeley, W. W. (2010). Network-level structural covariance in the developing brain. Proc. Natl. Acad. Sci. U.S.A. 107, 18191-18196. doi: 10.1073/pnas.1003 109107

Conflict of Interest Statement: The authors declare that the research was conducted in the absence of any commercial or financial relationships that could be construed as a potential conflict of interest.

Copyright (c) $2018 \mathrm{Li}, \mathrm{Li}$, Wang, Li and Li. This is an open-access article distributed under the terms of the Creative Commons Attribution License (CC BY). The use, distribution or reproduction in other forums is permitted, provided the original author(s) and the copyright owner are credited and that the original publication in this journal is cited, in accordance with accepted academic practice. No use, distribution or reproduction is permitted which does not comply with these terms. 